\title{
Generalized Dynamic Flow on Lossy Network
}

\author{
Santosh Gautam ${ }^{1}$, Urmila Pyakurel, Tanka Nath Dhamala \\ Central Department of Mathematics, Tribhuvan University, Kathmandu, Nepal \\ ${ }^{1}$ Corresponding author: yesgautam11@gmail.com
}

Received: Dec 6, 2018

Revised: Feb 28, 2019

Accepted: March 1, 2019

\begin{abstract}
Despite of implicit flow conservation on every arc of traditional network flow model, the generalized network flow model assumes the proportional and symmetric loss factor on each arc. This paper considers two terminal lossy networks specifying the portion of flow entering an arc at its tail node that reaches to its head node. We studied the generalized maximum continuous dynamic contraflow (GMCDCF) and generalized continuous earliest arrival contraflow (GCEACF) problems. The problems are efficiently solved with pseudo-polynomial time algorithms. Moreover, a fully polynomial time approximation scheme (FPTAS) is proposed in polynomial time.
\end{abstract}

Keywords: Generalized flow, lossy network, dynamic contraflow, approximation scheme

\section{Introduction}

As an inevitable part of disaster management, an evacuation planning is understood as a procedure of shifting evacuees from disaster (sources) areas to safety (sinks) areas as much as possible with minimum loss of life and properties. Due to a probable unfortunate death of evacuees, evacuees may not reach the destination with the same amount as departed [26,9]. To make the issues more realistic and real-life based, we generalized the flow model which maximizes the real amount reaching to destination in continuous time setting. The lossy network captures many generalized properties where flow value is only lost or conserved when these are sent from the sources to the sinks.

The static generalized flow problem was introduced by Dantzig [2] giving an LP formulation and can thus be solved in polynomial time. The combinatorial polynomial time algorithm was proposed in [7] and that has been improved later in [25]. Most of the polynomial-time algorithms for generalized maximum flow are developed and described in [26]. Authors in [13, 5, 9, 8] have presented efficient algorithms to solve this problem in static and dynamic networks. Gross and Skutella [9] proved that the generalized maximum dynamic flow (GMDF) model having both loss (gain) factors and transit time simultaneously is NP-hard. Also, we can find pseudopolynomial time algorithm that has been presented for GMDF and generalized maximum earliest 
arrival flow (GMEAF) problems in [9] on a lossy network in which the loss rate per time unit is identical on all arcs. Similarly, authors of $[16,17]$ studied the generalized dynamic contraflow on lossy network and extend the result for the FPTAS. Contraflow configuration is well-accepted model for evacuation planning which increase the outbound road capacities and reduce the evacuation time significantly. Authors in [12] showed that the evacuation time is increased at least 40 percent with at most 30 percent of the arc are reversals. This approach is important from the practical point of view. Some analytical solutions in different dynamic contraflow networks with discrete and continuous time can be found in [3, 12, 18-24].

Rest of the papers is planned as follows: In Section 2, we give mathematical formulation. Section 3 presents pseudo-polynomial time algorithms that solve the proposed problem. Approximate solution with FPTAS techniques are presented in Section 4 and finally Section 5 concludes the paper.

\section{Basic Denotations and Problem Formulation}

Let $N=(V, A, c, \lambda, \tau, s, d, T), 0<\lambda \leq 1$ be the generalized dynamic lossy network, where $V$ and $A$ denote the finite set of nodes and arcs with $n=|V|$ and $m=|A|$. The nodes $s$ and $d$ represent the source and the sink, respectively. The capacity $c(e) \in R^{+}$denotes the maximum amount of flow that may enter the arc $e \in A$ per time period and transit time $\tau(e) \in Z^{+}$gives the time needed to travel one unit of flow on $\operatorname{arc} e$. The set of outgoing and incoming arcs for node $i \in V$ are denoted as $A_{i}^{\text {out }}=\{(i, j) \in A\}$ and $A_{i}^{\text {in }}=\{(j, i) \in A\}, \forall j \in V$, respectively. We use $\mathbb{T}=[0, T)$ for the domain of continuous time interval. Here, each arc e $\in A$ has the proportional loss factor $\lambda(e) \equiv 2^{k \tau(e)}, k<0$, that is, in each time unit the same percentage of the remaining flow value is lost.

A generalized maximum continuous dynamic flow (GMCDF) is defined by a function $f: A \times$ $\mathbb{T} \rightarrow R^{+}$, which maximizes Equation (1) with respect to the constraints (2)-(4),

$$
\max \vartheta(f, T)=\int_{0}^{T} \sum_{e \in A_{d}^{i n}} \lambda(e) f(e, \sigma-\tau(e)) d \sigma
$$

such that:

$$
\begin{array}{cc}
\int_{0}^{T} \sum_{e \in A_{i}^{\text {in }}} \lambda(e) f(e, \sigma-\tau(e)) d \sigma=\int_{0}^{T} \sum_{e \in A_{i}^{\text {out }}} f(e, \sigma) d \sigma & \forall i \notin\{s, d\} \\
\int_{0}^{\xi} \sum_{e \in A_{i}^{\text {in }}} \lambda(e) f(e, \sigma-\tau(e)) d \sigma \geq \int_{0}^{\xi} \sum_{e \in A_{i}^{\text {out }}} f(e, \sigma) d \sigma & \forall i \notin\{s, d\}, \xi \in[0, T) \\
0 \leq f(e, \sigma) \leq c(e, \sigma) \quad \forall e \in A, \quad \sigma \in[0, T)
\end{array}
$$


Similarly, $f: A \times \mathbb{T} \rightarrow R^{+}$be the generalized maximum continuous earliest arrival flow (GMCEAF) function, which maximizes the flow value given by Equation (5) in every subinterval of the time $[\sigma, \sigma+\tau(e))$ with respect to the constraints (2)-(4),

$$
\max \vartheta(f, \sigma)=\int_{0}^{\xi} \sum_{e \in A_{d}^{i n}} \lambda(e) f(e, \sigma-\tau(e)) d \sigma \quad \forall e \in A, \quad \sigma, \xi \in[0, T)
$$

For the residual network $N^{R}=\left(V, A^{+} \cup A^{-}\right)$of $N$, each arc $e=(i, j) \in A$ has corresponding reverse arc $e^{-1}=(j, i)$. Similarly, the set $A^{+}$contains all arcs $e=(i, j)$ with static flow $g(e)<$ $c(e)$ and we assign a capacity of $c(e)-g(e)$, transit time $\tau(e)$ and loss factor $\lambda(e)$. The set $A^{-}$ contains all reverse arcs $e^{-1}=(j, i)$ with $g(e)>0$ and we assign a capacity of $c(e)$, transit time $-\tau(e)$ and loss factor $\frac{1}{\lambda(e)}$. The GMDF problem is solved using the time-expanded network [9] whose size depends on the time horizon $T$. Let $N_{T}=\left(V_{T}, A_{T}, \lambda_{T}, c_{T}\right)$ be the generalized timeexpanded network of $N$. Then, networks $N_{T}$ and $N$ send exactly the same amount of flow to the sink, $[9,8]$.

Standard flow decomposition simplifies the generalized flow by canceling the cycles which do not reach the destination. In contrast to the standard chain-decomposition, authors in $[10,11]$ introduce a nonstandard chain-decomposable flow that also works in a similar fashion. Recall that any network without flow-generating cycles can be turned into a lossy network.

Contraflow technique configures the given network as $\bar{N}=\left(V, E, c_{E}, \lambda, \tau, s, d, T\right)$, where corresponding data are defined as follows:

$c(\bar{e})=c(e)+c\left(e^{-1}\right)$ and $\tau(\bar{e})=\left\{\begin{array}{cc}\tau(e) & \text { if } e \in A \\ \tau\left(e^{-1}\right) & \text { otherwise, }\end{array}\right.$ for all $\bar{e} \in E$ in $\bar{N}$ if $e \vee \bar{e} \in A$ in $N$ and remaining graph structure and the data are unaltered. The modified loss factor is $\lambda(\bar{e}) \equiv 2^{k \tau(\bar{e})}$, $k<0$.

Natural transformation helps to transfer the discrete dynamic flow into a continuous dynamic flow and vice versa. Authors in [4] show the feasibility and continuity of the natural transformation. Authors define the flow rate $f(e)$ on arc $e$ in time interval $[\sigma, \sigma+1), \sigma \in Z^{+}$ and $f_{d}(e)$ on arc $e$ at time $\sigma \in Z^{+}$and prove that the total amount of flow is $f(e)$ in both situation, numerically: $f_{d}(e, \sigma)=f(e,[\sigma, \sigma+1))$. This transformation has the nice property that the amount of flow arriving at $j$ via arc $(i, j)$ in the unit time interval beginning at time $\sigma$ in the continuous flow will equal the amount of flow that arrives at $j$ via $(i, j)$ at time step $\sigma$ in the discrete flow. For the integral time horizon, natural transformation of chain decomposition flows is also feasible. If the time horizon $T$ is not integral, then we create a continuous $T$-horizon flow by natural transformation of discrete $\lceil T\rceil$-horizon chain decomposable flow into a continuous chain decomposable flow and stop sending flow along each chain flow $\gamma$ at time $T-\tau(\gamma)$ instead of time $\lceil T\rceil-\tau(\gamma)$. 


\section{Generalized Maximum Continuous Dynamic Contraflow Problem}

Generalization of maximum flow on continuous time setting has not been sufficiently studied before. There is no any strong polynomial time algorithm to solve GMCDF problem. Authors in [6] give a pseudo-polynomial time algorithm to solve Problem 1.

Problem 1: Given a network $N=(V, A, c, \lambda, \tau, s, d, T)$, the GMCDCF problem is to find a maximum continuous flow by maximizing objective (1) with respect to constraints (2)-(4) that can be sent from $\mathrm{s}$ to $\mathrm{d}$ within time $\mathrm{T}$, if the direction of arcs can be reversed at time zero.

To solve Problem (1), we converted the given network into auxiliary network using contraflow configuration. On the auxiliary network, we compute the GMDF using the algorithm of $[9,8]$. Then, we transform the discrete dynamic flow into the continuous flow by natural transformation of [4] in the same time complexity. We started with the zero flow, computed a maximum flow in the minimum loss path, augmented this flow and repeated this process until no $s-d$ path exists in the static residual network. It took pseudo-polynomial time to compute the GMCDCF on an auxiliary network.

Algorithm 1: Generalized maximum continuous dynamic contraflow (GMCDCF)

1. Given a network $N=(V, A, c, \lambda, \tau, s, d, T)$

2. Obtain the auxiliary network $\bar{N}=\left(V, E, c_{E}, \lambda, \tau, s, d, T\right)$ of $N$.

3. Compute the GMDF using the algorithm of $[9,8]$ on auxiliary network.

4. Transform discrete dynamic flow into the continuous dynamic flow using the natural transformation: $f_{d}(e, \sigma)=f(e,[\sigma, \sigma+1))$ for $\sigma \in[0, T),[4]$.

5. Arc $(j, i) \in A$ is reversed, if and only if the flow along arc $(i, j)$ is greater than $c(i, j)$ or if there is a nonnegative flow along arc $(i, j) \notin A$ and the resulting flow is GMCDCF with arc reversals on $N$.

For the optimality of Algorithm 1, we prove Lemma 1.

Lemma 1: The GMCDCF is less than or equal to the generalized maximum static contraflow (GMSCF) for the corresponding time-expanded network $\mathrm{N}_{T}$ of two terminal lossy network $N$.

Proof: Authors in $[16,18]$ show that every feasible flow in the GMDCF problem has an equivalent feasible flow to the GMSCF in the corresponding time-expanded network. Authors in [20] show that every feasible flow to the MCDCF problem has an equivalent feasible flow to the MSCF problem in the corresponding time-expanded network. Now, we conclude that every feasible flow to the GMCDCF problem has equivalent feasible GMSCF on the corresponding time-expanded network.

Theorem 1: The GMCDCF problem can be computed optimally by Algorithm 1 in two terminal lossy network $N=(V, A, c, \lambda, \tau, s, d, T)$. 
Proof: Steps 2 and 3 of Algorithm 1 are well defined and trivial. Step 4 is also feasible since the natural transformation converts the feasible $\lceil T\rceil$-horizon maximum discrete dynamic flow into the feasible $T$-horizon MCDF. Moreover, there is no any cycle flow on Step 3. Therefore, flow is either in an arc $(i, j)$ or $(j, i)$ but never in both arcs in Step 5. The flow is not greater than the reversed capacities on all the arcs at all-time units, thus, Step 5 is also feasible.

We proved the optimality. We know from feasibility that every feasible GMCDF solution of the MCDF problem in the $\bar{N}$ is feasible to the GMCDCF solution in the original network $N$.

$$
\bar{N}_{[G M C D F]} \leq N_{[G M C D C F]}
$$

where $\bar{N}_{[G M C D F]}$ and $N_{[G M C D C F]}$ represent the optimal flows on $\bar{N}$ and $N$, respectively.

Using Lemma 1, the GMSCF in $N_{T}$ and GMCDCF in $N$ has the following relation

$$
N_{[G M C D C F]} \leq N_{T[G M S C F]}
$$

As in $[1,24]$, the GMSCF in $N_{T}$ and GMSF in auxiliary $\bar{N}_{T}$ has the equivalent optimal outcome.

$$
N_{T[G S M C F]} \equiv \bar{N}_{T[G M S F]}
$$

Since the holdover flow is not allowed, the data for $\bar{N}$ are defined as follows. An auxiliary network of $N_{T}$ is $\bar{N}_{T}=\left(V_{T}, \bar{E}_{T}\right)$. The node set remains unaltered but the arc set is defined as:

$$
\bar{E}_{T}=\left\{\begin{array}{lc}
(i, j) & \text { if }(i, j) \in E_{T} \\
(j, i) & \text { otherwise }
\end{array}\right.
$$

Capacity function $\left(c_{\bar{E}_{T}}^{\tau}\right)$ is define as, $c_{\bar{E}_{T}}^{\tau}(\bar{e})=c_{E_{T}}^{\tau}(e)+c_{E_{T}}^{\tau}\left(e^{-1}\right)$ and the lost factor remains the same, $\bar{\lambda}(\bar{e}) \equiv 2^{k \tau(\bar{e})}, k<0, \forall \bar{e} \in \bar{E}_{T}$. Moreover, the GMDF in network $\bar{N}$ can be obtained by a temporally repeating chain flow of a generalized maximum flow in the time-expanded network $\bar{N}_{T}$.

$$
\bar{N}_{[G M D F]}=\bar{N}_{T[G M S F]}
$$

Applying the natural transformation of [4] for the Equation (9) and using above equations, we get

$$
N_{[G M C D C F]} \leq \bar{N}_{[G M C D F]}
$$

Combining inequalities (6) and (10) we have the optimum flow $N_{[G M C D C F]}=\bar{N}_{[G M C D F]}$.

Corollary 1: The two terminal GMCDCF problem can be solved in pseudo-polynomial time.

Proof: Steps 2 and 4 of Algorithm 1 are solved in linear time. A maxflow can be computed in $O(n m)$ time [15]. Thus, the time complexity of these steps is dominated by Step 3. The running time in Step3 is $O(\max f l o w \times T)[8,9]$. According to [4] discrete flow can convert in to continuous flow in the same time complexity. So, the complexity of Algorithm 1 is $O(n m \times T)$. 
Since, the complexity of maximum flow directly depends upon the time $T$, it is pseudopolynomial time.

Example 1: Consider the network $N$ in Fig. 1(a) to having a capacity, loss factor and transit times. Fig. 1(b) represents the corresponding auxiliary network. Fig. 1(c) represents the generalized continuous dynamic network and Fig. 1(d) gives the reconfigured network of $\bar{N}$ with minimum loss.

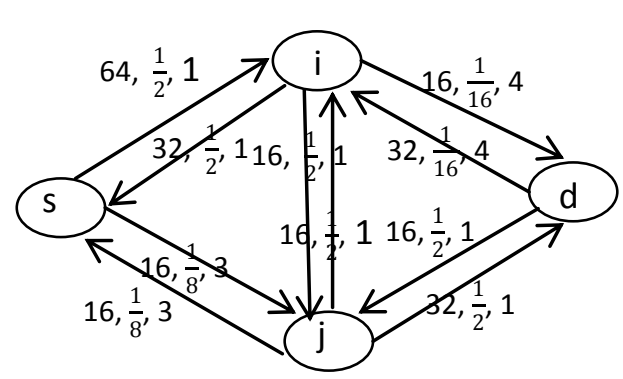

(a)

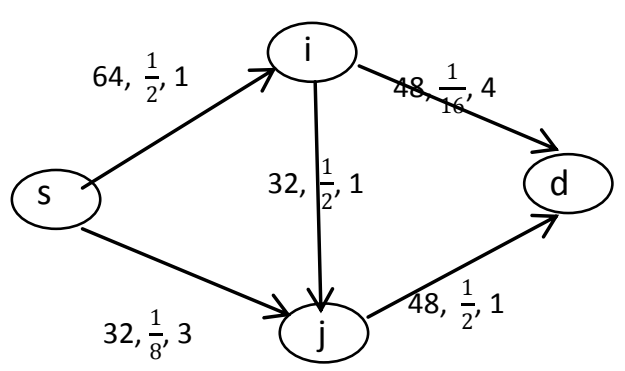

(c)

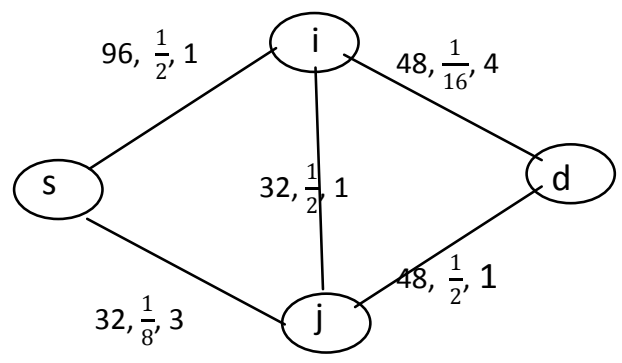

(b)

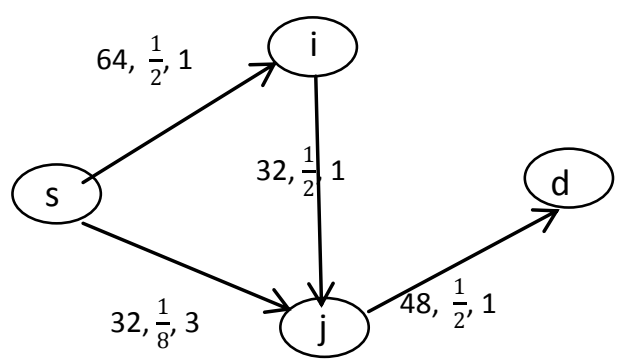

(d)

Fig.1: (a) Given lossy network $N$ (b) Auxiliary network $\bar{N}$ (c) Contraflow reconfiguration towards sink (d) Minimum loss network of $\bar{N}$

We apply Algorithm 1 to compute GMCDCF through minimum loss path. In Fig. 1(c), let us consider the following successive shortest paths on $\bar{N}$ :

$$
\begin{gathered}
P_{1}=s \rightarrow i \rightarrow d \text { with } \lambda\left(P_{1}\right)=\Pi_{e \in P_{1}} \lambda(e)=\frac{1}{2} \times \frac{1}{16}=\frac{1}{32} \\
P_{2}=s \rightarrow j \rightarrow d \text { with } \lambda\left(P_{2}\right)=\Pi_{e \in P_{2}} \lambda(e)=\frac{1}{8} \times \frac{1}{2}=\frac{1}{16} \\
P_{3}=s \rightarrow i \rightarrow j \rightarrow d \text { with } \lambda\left(P_{3}\right)=\Pi_{e \in P_{3}} \lambda(e)=\frac{1}{2} \times \frac{1}{2} \times \frac{1}{2}=\frac{1}{8} \\
P_{4}=s \rightarrow j \rightarrow i \rightarrow d \text { with } \lambda\left(P_{4}\right)=\Pi_{e \in P_{4}} \lambda(e)=\frac{1}{8} \times \frac{1}{2} \times \frac{1}{16}=\frac{1}{256}
\end{gathered}
$$


Since $\lambda\left(P_{4}\right)<\lambda\left(P_{1}\right)<\lambda\left(P_{2}\right)<\lambda\left(P_{3}\right)$ the path $P_{3}(s \rightarrow i \rightarrow j \rightarrow d)$ is the minimum loss path and path $P_{4}(s \rightarrow j \rightarrow i \rightarrow d)$ is the highest loss path according to the SSPA presented by Onaga $[13,14]$.

Now the Fig. 2 (a) and (b) give the GMCDCF with respective to Fig. 1 (c) and (d).
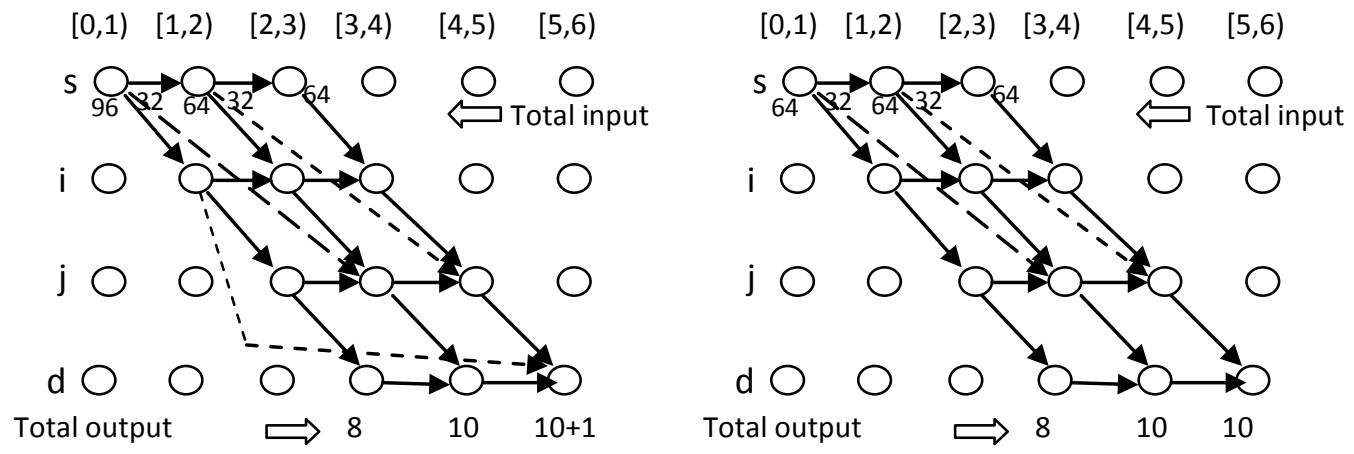

Fig. 2: Corresponding time-expanded network for Fig. 1(a) and (b)

\subsection{Maximum Continuous Earliest Arrival Contraflow Problem}

Earliest arrival flow ensures that the maximal number of evacuees is evacuated from the beginning of the process which is a most realistic approach to it. We define and solve Problem 2 in this section.

Problem 2: Given $N=(V, A, c, \lambda, \tau, s, d, T)$, the GCEACF problem computes the maximum flow that can be sent in every time period $0 \leq \xi \leq T$ from $\mathrm{s}$ to $d$ with arc reversal capability.

Lemma 2: Any GMCDCF solution induced by Algorithm 1 has the earliest arrival property.

Proof: Algorithm 1 gives an optimal solution of GMCDCF problem inducing temporally repeated flow on auxiliary network $\bar{N}$. However, authors [9] proved that the arrival pattern of GMDF without holdovers in intermediate nodes fulfills the earliest arrival property. Also, they decomposed the generalized flows into five types of elementary generalized flows on the basis of Onaga's optimality criterion [13]. The fact that the temporarily repeated GMCDCF obtained by this Algorithm has the earliest arrival flow property which is followed from the results of GEAF presented in $[8,9]$.

Corollary 2: The GMCDCF and GCEACF solutions are equivalent for two terminal lossy network.

Theorem 2: An optimal GCEACF on $N=(V, A, c, \lambda, \tau, s, d, T)$ can be obtained in pseudopolynomial time. 
GCEACF problem on two terminal lossy network has the same arrival property of GMCDCF problem. So, the time complexity for the GMCEAF problem has to be the same. Theorem 1 has proven the time complexity for GMCDCF is pseudo-polynomial.

\section{Approximate Generalized Maximum Dynamic Contraflow}

There is no any polynomial time algorithm to approximate the GMCDF solution without contraflow. We propose an FPTAS that computes an approximate solution of GMCDCF problem in a continuous time setting.

Theorem 3: $[8,9]$ There is neither a polynomial time algorithm nor a polynomial time approximation algorithm for the GMDF problem even on proportional gains, unless $\mathbb{P}=N P$.

Problem 3: Given $N=(V, A, c, \lambda, \tau, s, d, T)$ and $\epsilon>0$, does there exist an approximate GMCDCF solution with value at least within a factor $(1-\epsilon)$ of an optimal GMCDCF solution within time $T$ if the direction of the arcs can be reversed at time zero?

Algorithm 2 computes an approximate GMCDCF solution for Problem 3. It constructs an auxiliary network by contraflow configuration at time zero. In the configured network, capacity will increase but transit time and loss factor remain unaltered. Moreover, in Step 3, we use FPTAS $[9,8]$ to compute an approximate GMDF on auxiliary lossy network. Authors provide algorithms that have a running time polynomial in the input size of the problem and $\epsilon^{-1}$ and terminates after a polynomial number of iterations. In Step 4 we transferred the discrete dynamic flow into the continuous dynamic flow using natural transformation [4] in the same time complexity. There is no cycle with a positive flow in Step 3, thus Step 5 is well defined because the flow is either in are $(i, j)$ or $(j, i)$ only.

Algorithm 2: Approximate GMCDCF on lossy network

1. Given a lossy network $N=(V, A, c, \lambda, \tau, s, d, T)$.

2. Construct the auxiliary lossy network $\bar{N}=\left(V, E, c_{E}, \lambda, \tau, s, d, T\right), \epsilon>0$.

3. On $\bar{N}$, compute an approximate GMDF using an FPTAS of $[9,8]$.

4. Transform discrete dynamic flow into the continuous dynamic flow using the natural transformation: $f_{d}(e, \sigma)=f(e,[\sigma, \sigma+1))$ for $\sigma \in[0, T),[4]$.

5. Arc $(j, i) \in A$ is reversed, if and only if the flow along arc $(i, j)$ is greater than $\mathrm{c}(\mathrm{i}, \mathrm{j})$ or if there is a nonnegative flow along $\operatorname{arc}(i, j) \notin A$ and the resulting flow is GMCDCF with the arc reversals on $N$.

First, we prove following lemma to prove the optimality of Algorithm 2.

Lemma 3: For given $\epsilon>0$ and $U=\max _{\bar{e} \in E} b_{E}(\bar{e})$, let $O P T$ be the value of an optimal solution to the GMCDCF problem on $\bar{N}$. Algorithm 2 obtains a GMCDCF solution of value at least $O P T-\epsilon$, after all paths of length $\leq \tau^{*}$ where, 


$$
\tau^{*}=-\frac{1}{k}\left(\log \frac{1}{\epsilon}+\log m+\log U+2 \log T\right)
$$

Proof: We compute an $O P T$ solution for GMCDCF problem using our Algorithm 2 on two terminal network $\bar{N}$. Let $[i, i+1) \in[0, T)$ be the subinterval and $a_{i}$ be the amount of the flow sent into a path of length $i$. According to the natural transformation of [4] total flow amount $a_{i}$ on $[i, i+1)$ is same with discrete transit time $i$ (path of length $i$ ) in the time step $\sigma \in[0, T-i$ ) or $\sigma \in\{0,1, \ldots, T-i-1)$. Numerically: $f(e,[i, i+1))=f_{d}(e, i)$. According to the assumption the length of paths are monotonically increasing and there is no holdover. Therefore, the loss factor is $2^{k \cdot i}$ and the flow is sent into a path of length $i$ for $[T-i, T-i+1)$ interval. Suppose we have all paths of length $\leq i$ are found. Then, there remains only the path of length $i+1, \ldots, T$ intact. Now, the total flow reaches to the sink using the remaining paths that equals

$$
\int_{\omega=i+1}^{T} a_{\omega} \times 2^{k \omega} \times(T-\omega) d \omega
$$

For $k<0$, we have $\max \left\{2^{k(i+1)}, \ldots, 2^{k(T)}\right\}=2^{k(i+1)}$. Furthermore, it is clear that $T-\omega<T$. The total flow sent into the paths in one-time step is bounded by $m \times U$. This gives

$$
\int_{\omega=i+1}^{T} a_{\omega} \times 2^{k \omega} \times(T-\omega) d \omega \leq \int_{\omega=i+1}^{T} a_{\omega} \times 2^{k(i+1)} \times T d \omega \leq m \times U \times 2^{k(i+1)} \times T^{2}
$$

If we set $i=-\frac{1}{k}\left(\log \frac{1}{\epsilon}+\log m+\log U+2 \log T\right)-1$, then we get right hand side that equals $\epsilon$.

Lemma 4: For given $\epsilon>0$ and $U=\max _{\bar{e} \in E} b_{E}(\bar{e})$, let $O P T$ be the value of an optimal solution to the GMCDCF problem on $\bar{N}$. Then Algorithm 2 obtains a GMCDCF solution of value at least $(1-\epsilon) \times O P T$, after $\tau^{*}$-iterations where,

$$
\tau^{*}=-\frac{1}{k}\left[\left(\log \frac{1}{\epsilon}+\log m+\log U+2 \log T\right)\right]
$$

Proof: Using the argument and notation of Lemma 3, let $i_{0}$ be the length of the paths in the first iteration. We furthermore assume that the lossy network has the minimum capacity 1 , so that at least $2^{k i_{0}}$ flow is sent in the first iteration. The problem is to find $\tau^{*}$ such that the flow amount reached to the sink by later iterations is $\leq \epsilon \times O P T$. Then we obtain

$$
\int_{\omega=i_{0}+\tau^{*}}^{T} a_{\omega} \times 2^{k \omega} \times(T-\omega) d \omega \leq m \times U \times 2^{k\left(i_{0}+\tau^{*}\right)} \times T^{2} \leq \epsilon \times 2^{k i_{0}} \leq \epsilon \times O P T
$$

Now, from the above inequality, we can write, 


$$
m \times U \times 2^{k \tau^{*}} \times T^{2} \leq \epsilon \Leftrightarrow \tau^{*} \geq-\frac{1}{k}\left[\left(\log \frac{1}{\epsilon}+\log m+\log U+2 \log T\right)\right]
$$

Based on these premises, the lemma is concluded.

Theorem 4: Algorithm 2 solves the approximate GMCDCF problem optimally.

Proof: We reverse the directions of arcs from sources to sinks in Step 2. According to contraflow configuration, transit time and unaltered loss factor do not influence the feasibility. So that Step 2 is feasible. As the FPTAS introduced by $[9,8]$ is feasible, Step 3 is also feasible. In Step 3, there is no any cycle with a positive flow so that flow in Step 5 is either along arc $(i, j)$ or $(j, i)$ but

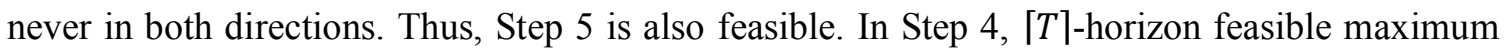
discrete dynamic flow is converted into $T$-horizon feasible maximum continuous dynamic flow by natural transformation [4]. So, Step 4 is also feasible. Thus, Algorithm 2 is feasible.

For the optimality of the Algorithm 2, based on the feasibility condition, every feasible approximate GMDF in the auxiliary network $\bar{N}$ is feasible to the approximate GMDCF solution of the network $N$. Also every feasible discrete dynamic flow and continuous dynamic flow has the same time complexity with equivalent output [4]. The amount of flow obtained in Step 3 cannot be changed in Step 4, the resulting flow is an optimal solution to the approximate GMDCF. Thus, the approximate GMCDF solution in $\bar{N}$ is equal to the approximate GMCDCF solution on $N$.

Corollary 4: For given $\epsilon>0, U=\max _{\bar{e} \in E} b_{E}(e)$ and $k<0$ on $N$, Algorithm 2 solves the approximate GMCDCF problem in $O\left(\operatorname{maxflow} \times\left(\log \epsilon^{-1}+\log U+\log T\right)\right)$ time.

\section{Conclusion}

Flow models with flow conservation constraints may not address the real flow phenomena in some practical scenarios. The generalization of flow models with loss factor attached is proximate to reflect and resolve the real world situation more accurately. The continuous time approach gives more accurate results in spite of the higher computational complexity. Naturally, the nonexistence of integrality in solution makes the computation more challenging. In this paper, we study the GMCDCF and GCEACF problems and also provide the algorithms of pseudo-polynomial time complexity. While the generalized maximum flow containing loss factor and transit time simultaneously on each arc cannot be solved in polynomial time, unless $P=N P$ holds, we have presented an FPTAS for the special case of a lossy network with proportional loss factors. To the best of our knowledge, these problems in a continuous time setting are introduced and solved for the first time.

Acknowledgment: The first author acknowledges the DAAD support under the partnership program between the departments of mathematics at Tribhuvan Unversity, Mindanao State University IIT and the University of Kaiserslautern, and that of University Grants Commission, Nepal. The second and third authors thank for the support of AvH Foundation. 


\section{References}

[1] Arulselvan A (2009), Network model for disaster management, PhD Thesis, University of Florida.

[2] Dantzig EW (1962), Linear programming and extensions, Princeton University Press.

[3] Dhamala TN, Pyakurel U and Dempe S (2018), A critical survey on the network optimization algorithms for evacuation planning problems, International Journal of Operations Research (TW), 15(3): 101-133.

[4] Fleischer LK and Tardos E (1998), Efficient continuous time dynamic network flow algorithms, Operations Research Letters, 23: 71-80.

[5] Fleischer LK and Wayne KD (2002), Fast and simple approximation schemes for generalized flow. Mathematical programming, 91: 215-238.

[6] Gautam S and Pyakurel U (2018), Generalized maximum continuous dynamic contraflow on lossy network, Extended Abstract, APORS 2018, Nepal, 11: 128-130.

[7] Goldberg AV, Plotkin SA and Tardos E (1991), Combinatorial algorithms for the generalized circulation problems. Mathematics of Operations Research, 16: 351-379.

[8] Gross M (2014), Approximation algorithms for complex network flow over time problems, $\mathrm{PhD}$ Thesis, Technical University, Berlin, Germany.

[9] Gross M and Skutella M (2012), Generalized maximum flows over time, Approximation and Online Algorithms Lecture Notes in Computer Science. 7164: 247-260.

[10] Hoppe B and Tardos E (2000), The quickest transshipment problem, Mathematics of Operations Research, 25: 36-62.

[11] Hoppe BE (1995), Efficient dynamic network flow algorithms, $\mathrm{PhD}$ thesis, Cornell University.

[12] Kim S, Shekhar S and Min M (2008), Contraflow transportation network reconfiguration for evacuation route planning, IEEE Transactions on Knowledge and Data Engineering, 20: 1-15.

[13] Onaga K (1966), Dynamic programming of optimum flows in lossy communication nets, IEEE Transactions on Circuit Theory, 13: 282-287.

[14] Onaga K (1967), Optimal flows in general communication networks, Journal of the Franklin Institute, 282(4): 308-327.

[15] Orlin JB (2013), Maxflow in $O(\mathrm{~nm})$ time, or better, Proceedings of the 45th ACM Symposium on the Theory of Computing, 13: 765-774.

[16] Pyakurel U (2016), Evacuation planning problem with contraflow approach, PhD Thesis, IOST, Tribhuvan University.

[17] Pyakurel U (2018), Generalized contraflow for evacuation planning problem, Journal of Nepal Mathematical Society, 1: 38-49.

[18] Pyakurel U, Hamacher HW and Dhamala TN (2014), Generalized maximum dynamic contraflow on lossy network. International Journal of Operations Research Nepal, 3: 27-44.

[19] Pyakurel U and Dhamala TN (2016), Continuous time dynamic contraflow models and algorithms, Advanced in Operations Research, Volume 2016: 1-6.

[20] Pyakurel U and Dhamala TN (2017), Continuous dynamic contraflow approach for evacuation planning, Annals of Operations Research, 253: 573-598.

[21] Pyakurel U, Nath HN and Dhamala TN (2018), Efficient contraflow algorithms for quickest evacuation planning, Science China Mathematics, DOI 10.1007/s11425-017-9264-3, 61(11): 2079-2100. 
[22] Pyakurel U, Nath HN and Dhamala TN (2018), Partial contraflow with path reversals for evacuation planning, Annals of Operations Research, DOI 10.1007/s10479-018-3031-8.

[23] Pyakurel U, Dhamala TN and Dempe S (2017), Efficient continuous contraflow algorithms for evacuation planning problems, Annals of Operations Research, 61: 2079-2100.

[24] Rebennack S, Arulselvan A, Elefteriadou L and Pardalos PM (2010), Complexity analysis for maximum flow problems with arc reversals, Journal of Combinatorial Optimization, 19: 200216.

[25] Radzik T (1998), Faster algorithms for the generalized network flow problem, Mathematics of Operations Research, 23: 69-100.

[26] Wayne KD (1999), Generalized maximum flow algorithms, PhD Thesis, Cornell University. 